\title{
Unsteady MHD Mixed Convection Flow past a Vertical Porous Plate in Presence of Radiation
}

\author{
S. Sivaiah ${ }^{1}$, G. Murali ${ }^{2}$, M. C. K Reddy ${ }^{3}$ and R. Srinivasa Raju ${ }^{4}$ \\ ${ }^{1}$ Department of Mathematics, GITAM University, Hyderabad, India. \\ ${ }^{2}$ Department of Mathematics, Rayalaseema University, Kurnool, India. \\ ${ }^{3}$ Department of Mathematics, Osmania University, Hyderabad, India. \\ ${ }^{4}$ Department of Mathematics, BVRIT, Narsapur, India. \\ E-mail: saihanuman79@yahoo.com
}

\begin{abstract}
This study examines the problem of unsteady MHD mixed convective flow past a vertical porous plate in presence of radiation. The coupled non linear partial differential equations are solved numerically by a finite element method. The effects of the material parameters on the velocity, temperature and concentration fields are shown graphically.
\end{abstract}

Keywords: MHD Mixed Convection, Radiation, FEM.

\section{Introduction}

In recent years, the flows of fluid through porous media are of principal interest because these are quite prevalent in nature. Such flows have attracted the attention of a number of scholars due to their application in many branches of science and technology, viz., in the field of agriculture engineering to study the underground water resources, seepage of water in riverbeds, in petroleum technology to study the movement of natural gas, oil and water through oil reservoirs, in chemical engineering for filtration and purification processes. The convection problem in porous medium has also important applications in geothermal reservoirs and geothermal energy extractions. A comprehensive review of the studies of convective heat transfer mechanism through porous media has been made by Nield and Bejan [1]. Hiremath and Patil [2] studied the effect on free convection currents on the oscillatory flow through a porous medium, which is bounded by vertical plane surface of constant temperature. Fluctuating heat and mass transfer on three-dimensional flow through a porous medium with variable permeability 
has been discussed by Sharma et al. [3]. Hydromagnetic convection with heat and mass transfer in porous medium has been studied due to its importance in the design of Magnetohydrodynamics (MHD) generators and accelerators in geophysics, design of ground water system, energy storage system, soilsciences, astrophysics, nuclear power reactors and so on. Magnetohydrodynamics is currently undergoing a period of great enlargement and differentiation of subject matter. The interest in these new problems generates from their importance in liquid metals, electrolytes and ionized gases. Unsteady hydromagnetic free convection flow of Newtonian fluid has been investigated by Helmy [4].Chaudhary and Sharma [5] considered combined heat and mass transfer by laminar mixed convection flow from a vertical surface with induced magnetic field. Hydromagnetic unsteady mixed convection and mass transfer flow past a vertical porous plate immersed in a porous medium was investigated by Sharma and Chaudhary [6]. El-Amin [7] considered the MHD free-convection and mass transfer flow in a micro polar fluid over a stationary vertical plate with constant suction. Combined heat and mass transfer problems with chemical reaction are of importance in many processes and have, therefore, received a considerable amount of attention in recent years. In processes such as drying, evaporation at the surface of water body, energy transfer in wet cooling tower and the flow in a desert cooler, heat and mass transfer occur simultaneously. Chemical reaction can be codified as either homogeneous or heterogeneous processes. A homogeneous reaction is one that occurs uniformly through a given phase. In contrast, a heterogeneous reaction takes place in a restricted region or within the boundary of a phase. A reaction is said to be first order, if the rate of reaction is directly proportional to the concentration itself which has many applications in different chemical engineering processes and other industrial applications such as polymer production, manufacturing of ceramics or glassware and food processing Cussler [8].Das et al. [9] considered the effects of first order chemical reaction on the flow past an impulsively started infinite vertical plate with constant heat flux and mass transfer.Muthucumarswamy and Ganesan [10] and Muthucumarswamy[11] studied first order homogeneous chemical reaction on flow past infinite vertical plate.

In the above mentioned studies the effects of heat sources/sinks and radiation have not been considered. Due to its great applicability to ceramic tiles production problems, the study of heat transfer in the presence of a source/sink has acquired newer dimensions. Actually, many processes in new engineering areas occur at high temperature and knowledge of radiation heat transfer becomes imperative for the design of the pertinent equipment. Nuclear power plants, gas turbines and the various propulsion devices for aircraft, missiles, satellites, and space vehicles are examples of such engineering areas. Kandasamy et al. [12] discussed heat and mass transfer effect along a wedge with heat source and concentration in the presence of suction/injection taking into account the chemical reaction of first order. Sharma et al.[13,14] have reported on the radiation effect with simultaneous thermal and mass diffusion in MHD mixed convection flow from a 
vertical surface.Sharma et al. $[15,16]$ discussed radiation effect on free convective flow along a uniform moving porous vertical plate in the presence of heat source/sink and transverse magnetic field.

Israel Cookey et al.[17] investigated the influence of viscous dissipation and radiation on unsteady MHD free convection flow past an infinite heated vertical plate in porous medium with time dependent suction.Gebhar [18] shown the importance of viscous dissipative heat in free convection flow in the case of isothermal and constant heat flux in the plate. Soundalgekar [19] analyzed the effect of viscous dissipative heat on the two dimensional unsteady, free convective flow past an vertical porous plate when the temperature oscillates in time and there is constant suction at the plate.

In spite of all these studies, the unsteady MHD mixed convictive flow past a vertical porous plate in presence of radiation has received little attention. The dimensionless equations are solved by using the finite element method. The effects of various governing parameters on the velocity, temperature and concentration are shown in figures and discussed in detail.

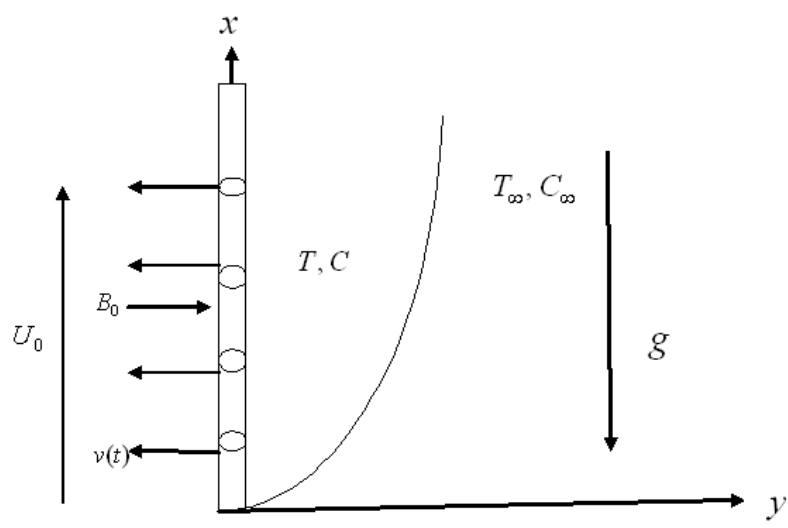

Fig.1. Flow Configuration and Coordinate system

\section{Mathematical Analysis}

An unsteady two-dimensional flow of an incompressible and electrically conducting viscous fluid, along an infinite vertical porous flat plate embedded in a porous medium is considered. The $x$-axis is taken on the infinite plate, and parallel to the free-stream velocity which isvertical and the $y$-axis is taken normal to the plate. A magnetic field $B_{0}$ of uniform strength isapplied transversely to the direction of the flow. Initially the plate and the fluid are at sametemperature $T_{\infty}$ in a stationary condition with concentration level $C_{\infty}$ at all points. For $t>0$, the plate starts moving impulsively in its own plane with a velocity $U_{0}$, its temperature israised to $T_{w}$ and the concentration level at the plate 
is raised to $C_{w}$. The flow configuration and coordinate system are shown in Fig. 1.The fluid is assumed to have constant properties except that the influence of the density variations with temperature and concentration, which are considered only in the body force term. Under the above assumptions, the physical variables are functions of $y$ and $t$ only. Assuming that the Boussinesq and boundary layer approximation hold and using the Darcy-Forchheimer model, the basic equations, which govern the problem, are given by:

$$
\begin{aligned}
& \frac{\partial v}{\partial y}=0 \\
& \frac{\partial u}{\partial t^{\prime}}+v \frac{\partial u}{\partial y}=v \frac{\partial^{2} u}{\partial y^{2}}+g \beta\left(T-T_{\infty}\right)+g \beta^{*}\left(C-C_{\infty}\right)-\frac{\sigma B_{0}^{2}}{\rho} u-\frac{v}{K} u-\frac{b}{K} u^{2} \\
& \frac{\partial T}{\partial t^{\prime}}+v \frac{\partial T}{\partial y}=\alpha \frac{\partial^{2} T}{\partial y^{2}}-\frac{1}{\rho c_{p}} \frac{\partial q_{r}}{\partial y}+\frac{v}{c_{p}}\left(\frac{\partial u}{\partial y}\right)^{2} \\
& \frac{\partial C}{\partial t}+v \frac{\partial C}{\partial y}=D_{m} \frac{\partial^{2} C}{\partial y^{2}}-k_{r}\left(C-C_{\infty}\right)
\end{aligned}
$$

By using the Rosseland approximation, the radiative heat flux $q_{r}$ is given by

$$
q_{r}=-\frac{4 \sigma_{s}}{3 k_{e}} \frac{\partial T^{4}}{\partial y}
$$

Where $u, v$ are the Darcian velocity components in the $x$ - and $y$-directions respectively, $t$ is the time, $v$ is the kinematic viscosity, $g$ is the acceleration due to gravity, $\rho$ is the density, $\beta$ is the coefficient of volume expansion, $\beta^{*}$ is the volumetric coefficient of expansion with concentration, $K$ is the Darcy permeability, $b$ is the empirical constant, $B_{0}$ is the magneticinduction, $T$ and $T_{\infty}$ are the temperature of the fluid inside the thermal boundary layer andthe fluid temperature in the free stream, respectively, while $C$ and $C_{\infty}$ are the corresponding concentrations. Also, $\sigma$ is the electric conductivity, $\alpha$ is the thermal diffusivity, $\sigma_{s}$ is the Stefan-Boltzmann constant and $k_{e}$ - the mean absorption coefficient. $D_{m}$ is the coefficient of mass diffusivity, $c_{p}$ is the specific heat at constant pressure, $q_{r}$ is the Radiative heat flux in the $y$-direction, $k_{r}$ is chemical reaction parameter.Initially $(t=0)$ the fluid and the plate are at rest.

Thus the no slip boundary conditions at the surface of the plate for the above problem for $t>0$ are: 


$$
\begin{array}{llllrlrl}
u=U_{0}, & T & =T_{w}, & C=C_{w} & & \text { at } & & y=0 \\
u & =0, & T & =T_{\infty}, & C=T_{\infty}, & \text { as } & & y \rightarrow \infty
\end{array}
$$

Now in order to obtain non-dimensional partial differential equations we are introducing

following dimensionless variables and constants.

$$
\begin{aligned}
& U=\frac{u}{u_{0}}, \quad Y=\frac{u_{0} y}{v}, \quad t=\frac{t^{\prime} u_{0}}{v}, \theta=\frac{T-T_{\infty}}{T_{w}-T_{\infty}}, \phi=\frac{C-C_{\infty}}{C_{w}-C_{\infty}}, \quad G r=\frac{\nu \beta g\left(T_{w}-T_{\infty}\right)}{u_{0}^{3}}, \\
& G m=\frac{v \beta^{*} g\left(C_{w}-C_{\infty}\right)}{u_{0}^{3}}, \quad \operatorname{Pr}=\frac{v}{\alpha}, \quad S c=\frac{v}{D m}, M=\frac{\sigma B_{0}^{2} v}{\rho u_{0}^{2}}, E c=\frac{u_{0}^{2}}{c_{p}\left(T_{w}-T_{\infty}\right)}, \\
& R=\frac{K_{T} K^{*}}{4 \sigma_{r} T_{\infty}^{3}}, \quad D a=\frac{K u_{0}^{2}}{v^{2}}, F s=\frac{b u_{0}}{v}, \quad K^{2} r=\frac{k_{r} v}{u_{0}^{2}},
\end{aligned}
$$

By introducing above dimensionless variables and constants the equations (2) (3) (4) converted as follows

$$
\begin{aligned}
& \frac{\partial U}{\partial t}-\frac{\partial U}{\partial Y}=\frac{\partial^{2} U}{\partial Y^{2}}+G r \theta+G m C-M U-\frac{1}{D a} U-\frac{F s}{D a} U^{2} \\
& \frac{\partial \theta}{\partial t}-\frac{\partial \theta}{\partial Y}=\frac{1}{\operatorname{Pr}}\left[1+\frac{4}{3 R}\right] \frac{\partial^{2} \theta}{\partial Y^{2}}+E c\left(\frac{\partial U}{\partial Y}\right)^{2} \\
& \frac{\partial \phi}{\partial t}-\frac{\partial \phi}{\partial Y}=\frac{1}{S c} \frac{\partial^{2} \phi}{\partial Y^{2}}-K^{2} r \phi
\end{aligned}
$$

Where $G r, G m, M, D a, F s, \operatorname{Pr}, R, E c, S c$ and $K r$ are the thermal Grashof number, SolutalGrashof number, Magnetic parameter, Local Darcy number, Local Forchheimer number,Prandtl number, thermal radiation, Eckert number, Schmidt number and chemical reaction parameter respectively.

The corresponding boundary conditions for $t>0$

$$
\begin{aligned}
& U=1, \quad \theta=1, \quad \phi=1 \quad \text { at } \quad Y=0 \\
& U=0, \quad \theta=0, \quad \phi=0 \quad \text { as } \quad Y \rightarrow \infty
\end{aligned}
$$

\section{Solution of the Problem}

By applying Galerkin finite element method for equation (8), (9) and (10) over the element $(e),\left(y_{j} \leq y \leq y_{k}\right)$ are becomes: 


$$
\begin{aligned}
& \int_{y_{j}}^{y_{k}} N^{(e)^{T}}\left[\frac{\partial^{2} U^{(e)}}{\partial y^{2}}+\frac{\partial U^{(e)}}{\partial y}-\frac{\partial u^{(e)}}{\partial t}-N U^{(e)}+P_{1}\right] d y=0 \\
& \int_{y_{j}}^{y_{k}} N^{(e)^{T}}\left[\left(1+\frac{4}{3 R}\right) \frac{\partial^{2} \theta^{(e)}}{\partial y^{2}}+\operatorname{Pr} \frac{\partial \theta^{(e)}}{\partial y}-\operatorname{Pr} \frac{\partial \theta^{(e)}}{\partial t}+P_{2}\right] d y=0 \\
& \int_{y_{j}}^{y_{k}} N^{(e)^{T}}\left[\frac{\partial^{2} C^{(e)}}{\partial y^{2}}+P S c \frac{\partial C^{(e)}}{\partial y}-S c \frac{\partial C^{(e)}}{\partial t}-S c K_{r} C^{(e)}\right] d y=0 \\
& \text { where } \quad P_{1}=G r \theta+G m \phi, N=\left(M+\frac{1}{D a}+\frac{F s}{D a}\right), \quad P_{2}=\operatorname{Pr} E c\left(\frac{\partial u}{\partial y}\right)^{2}
\end{aligned}
$$

Let the linear piecewise approximation solution

$$
\begin{array}{r}
U^{(e)}=N_{j}(y) U_{j}(t)+N_{k}(y) U_{k}(t)=N_{j} U_{j}+N_{k} U_{k} \\
\theta^{(e)}=N_{j}(Y) \theta_{j}(t)+N_{k}(Y) \theta_{k}(t)=N_{j} \theta_{j}+N_{k} \theta_{k} \\
\phi^{(e)}=N_{j}(Y) \phi_{j}(t)+N_{k}(Y) \phi_{k}(t)=N_{j} \phi_{j}+N_{k} \phi_{k}
\end{array}
$$

Where $N_{j}=\frac{Y_{k}-Y}{Y_{k}-Y_{j}}, N_{k}=\frac{Y-Y_{j}}{Y_{k}-Y_{j}}$

$$
N^{(e)^{T}}=\left[\begin{array}{ll}
N_{j} & N_{k}
\end{array}\right]^{T}=\left[\begin{array}{l}
N_{j} \\
N_{k}
\end{array}\right]
$$

The set of differential Equations (8) to (10) subject to the boundary conditions (11) are highly nonlinear, coupled and therefore it cannot be solved analytically. Hence, following Reddy [20] and Bathe [21] the finite element method is used to obtain an accurate and efficient solution to the boundary value problem under consideration. The fundamental steps comprising the method are as follows:

Step 3.1: Discretization of the domain into elements:

The whole domain is divided into finite number of sub-domains, a process known as discretization of the domain. Each sub-domain is termed a finite element. The collection of elements is designated the finite element mesh.

Step 3.2: Derivation of the element equations:

The derivation of finite element equations i.e. algebraic equations among the unknown parameters of the finite element approximation, involves the following three steps: 
a. Construct the variational formulation of the differential equation.

b. Assume the form of the approximate solution over a typical finite element.

c. Derive the finite element equations by substituting the approximate solution into variational formulation.

Step 3.3: Assembly of element equations:

The algebraic equations so obtained are assembled by imposing the inter-element continuity conditions. This yields a large number of algebraic equations, constituting the global finite element model, which governs the whole flow domain.

Step 3.4: Impositions of boundary conditions:

The physical boundary conditions defined in equation (11) are imposed on the assembled equations.

Step 3.5: Solution of the assembled equations:

The final matrix equation can be solved by a direct or indirect (iterative) method. For computational purposes, the coordinate $y$ is varied from 0 to $y_{\max }=4$, where $y_{\max }$ represents infinity i.e. external to the momentum, energy and concentration boundary layers. The whole domain is divided into a set of 200 line elements of equal width 0.05 , each element being three noded. Thus after assembly of all the elements equations we obtain a matrix of order $402 \times 402$. This system of equations as obtained after assembly of the elements equations is non-linear therefore an iterative scheme has been used to solve it. The system is linearized by incorporating known functions. After applying the given boundary conditions only a system of 195 equations remains for the solution which has been solved using Gauss elimination method. This process is repeated until the desired accuracy of 0.0005 is obtained.

\section{Results and Discussion}

In the preceding sections, the problem of unsteady MHD mixed convictive flow past a vertical porous plate in presence of radiation was formulated and the dimensionless governing equations were solved by means of a finite element method. In the present study we adopted the following default parameter values of finite element computations: $\quad G r=2.0, G m=2.0, \quad M=0.3, D a=0.5$, $F s=0.09, \operatorname{Pr}=0.71, R=0.5, E c=0.5, \quad S c=0.22, K r=0.5 \quad$ All graphs therefore correspond to these values unless specifically indicated on the appropriate graph.

The influence of the Grashof number on the velocity is presented in Fig .2. Increase in the Grashof number contributes to the increase in velocity when all other parameter that appears in the velocity field are held constant The influence of the modified Grashof number on the velocity is presented in Fig.3. It is 
observed that, while all other parameters are held constant and velocity increases with an increase in modified Grashof number.

For different values of the Prandtl number $\operatorname{Pr}$, the velocity and temperature profiles are plotted in Figs. 4 and 5. The Prandtl number defines the ratio of momentum diffusivity to thermal diffusivity. From Fig. 4, it is clear that an increase in the Prandtl number leads to a fall in the velocity. From Fig.5, it is observed that an increase in the Prandtl number results a decrease of the thermal boundary layer thickness and in general lower average temperature within the boundary layer. The reason is that smaller values of $\operatorname{Pr}$ are equivalent to increasing the thermal conductivities, and therefore heat is able to diffuse away from the heated surface more rapidly than for higher values of $\operatorname{Pr}$. Hence in the case of smaller Prandtl numbers as the boundary layer is thicker and the rate of heat transfer is reduced.

For different values of radiation parameter $R$, the velocity profiles are plotted in Fig.6. Here, as the value of $R$ increases the velocity decreases, with an increasing in the flow boundary layer thickness. Thus, thermal radiation enhances convective flow. The effects of radiation parameter $R$ on the temperature profiles are presented in Fig.7. From this figure we observe that, as the value of $R$ increases the temperature profiles decreases, with an increasing in the thermal boundary layer thickness.

The effects of the viscous dissipation parameter i.e., the Eckert number $E c$ on the velocity and temperature are shown in Figs.8 and 9. Greater viscous dissipative heat causes a rise in the temperature as well as the velocity profiles. Eckert number $E c$ designates the ratio of the kinetic energy of the flow to the boundary layer enthalpy difference. The effect of viscous dissipation on the flow field is to increase the energy, yielding a greater fluid temperature and as a consequence greater buoyancy force. The increase in the buoyancy force due to an increase in the dissipation parameter enhances the convective velocity and also the temperature.

Figs. 10 and 11 illustrate the velocity and concentration profiles for different values of Schmidt number $S c$. The Schmidt number embodies the ratio of the momentum to the mass diffusivity. The Schmidt number therefore quantifies the relative effectiveness of momentum and mass transport by diffusion in the hydrodynamic (velocity) and concentration (species) boundary layers. It is observed that as the Schmidt number increases the concentration decreases. This causes the concentration buoyancy effects to decrease yielding a reduction in the fluid velocity. The reductions in the velocity and concentration profiles are accompanied by simultaneous reductions in the velocity and concentration boundary layers.

The influences of chemical reaction parameter $K r$ on the velocity profiles across the boundary layer are presented in Fig.12. We see that the velocity distribution across the boundary layer decreases with increasing of $K r$. For different values of the chemical reaction parameter $K r$, the concentration profiles plotted in Fig.13. It 
is obvious that the influence of increasing values of $K r$, the concentration distribution across the boundary layer decreases.

\section{Conclusions}

In this paper we have studied the unsteady MHD mixed convictive flow past a vertical porous plate in presence of radiation. The non- dimensional governing equations are solved with the help of finite element method. The conclusions of the study are as follows:

5.1 The velocity increases with the increase Grashof number and modified Grashof number.

5.2 An increase in the prandtl number decreases the velocity and temperature.

5.3 With increase in Radiation the velocity and temperature profiles are decreasing.

5.4 With increase in Eckert number is shown to reduce the velocity and temperatures in the flow.

5.5 The velocity as well as concentration decreases with an increase in the Schmidt number.

5.6 The velocity as well as concentration decreases with an increase in the chemical reaction parameter.

\section{References}

[1] Nield, D.A., and Bejan, A.1998. Convection in porous media. 2nd edition, Springer-Verlag, Berlin.

[2] Hiremath, P.S., and Patil, P.M. 1993. Free convection effects on oscillatory flow of couple stress field through a porous medium. Acta Mech., 98: 143158.

[3] Sharma, B. K., Chaudhary, R.C., and Sharma, P.K. 2007. Fluctuating mass transfer on three dimensional flow through a porous medium with variable permeability. Advances in Theoretical and Applied Mathematics., 2(3): 257267.

[4] Helmy, K.A. 1998. MHD unsteady free convection flow past a vertical porous plate, ZAMM., 98: 255-270.

[5] Chaudhary, R.C., and Sharma, B.K. 2006. Combined heat and mass transfer by laminar mixed convection flow from a vertical surface with induced magnetic field. Journal of Applied Physics., 99: 34901-10. 
[6] Sharma, B.K., and Chaudhary, R.C. 2008. Hydromagnetic unsteady mixed convection and mass transfer flow past a vertical porous plate immersed in a porous medium with Hall effect. Engineering Transactions., 56(1): 3-23.

[7] El-Amin, M.F. 2001. Magnetohydrodynamic free convection and mass transfer flow in micropolar fluid with constant suction. J. Magn. Mater., 234: 567-574.

[8] Cussler, E.L. 1998. Diffusion mass transfer in fluid systems. 2nd ed. Cambridge University Press.

[9] Das, U.N., Deka, R.K., and Soundalgekar, V.M. 1994. Effects of mass transfer on flow past an impulsively started infinite vertical plate with constant heat flux and chemical reaction, Forschung im Engenieurwesen Engineering Research Co., 284-287.

[10] Muthucumarswamy, R., and Ganesan, P. 2001. First order chemical reaction on flow past an impulsively started vertical plate with uniform heat and mass flux. Acta., Mech. 147: 45-57.

[11] Muthucumarswamy, R. 2002. Effects of a chemical reaction on moving isothermal vertical surface with suction. Acta. Mech., 155: 65-70.

[12] Kandasamy, Periasamy, R.K., and Sivagnana, K.K.P. 2005. Effects of chemical reaction, heat and mass transfer along wedge with heat source and concentration in the presence of suction or injection. Int. J. Heat Mass transfer., 48: 1388-1394.

[13] Sharma, B.K., Jha, A.K., and Chaudhary, R.C. 2006. Radiation effect with simultaneous thermal and mass diffusion in MHD mixed convection flow from a vertical surface with Ohmic heating. Romania Journal of Physics., 51(7-8): 715-727.

[14] Sharma, B.K., Agarwal, M.,and Chaudhary, R.C. 2006. MHD fluctuating free convective flow with radiation embedded in porous medium having variable permeability and heat source/sink. Journal of Technical Physics., 47(1): 47-58.

[15] Sharma, B.K., Chaudhary, R.C., and Agarwal, M. 2007. Radiation effect on temperature distribution in three-dimensional Couette flow with injection or suction. Applied Mathematics and Mechanics., 28(3): 309-316.

[16] Sharma, B.K., Chaudhary, R.C., and Agarwal, M. 2008. Radiation effect on steady free convective flow along a uniform moving porous vertical plate in presence of heat source/sink and transverse Magnetic Field. Bull. Cal. Math. Soc., 100: 529-538.

[17] Israel-Cookey, C., Ogulu, A., and Omubo-Pepple, V.B. (2003). Influence of Viscous Dissipation on Unsteady MHD free Convection Flow Past an Infinite Heated Vertical Plate in Porous Medium with Time Dependent Suction, International Journal of Heat and Mass Transfer., 46: 2305-2311.

[18] Gebhar, B. 1962. Effects of Viscous Dissipative in Natural Convection, Journal of Fluid Mechanics., 14: 225-232. 
[19] Soundalgekar, V. M. 1972. Viscous Dissipative Effects on Unsteady free Convective Flow Past an Vertical Porous Plate with Constant Suction, International Journal of Heat and Mass Transfer., 15:1253-1261.

[20] Reddy , J.N. 1985. An Introduction to the Finite Element Method. McGrawHill, New York.

[21] Bathe, K.J. 1996. Finite Element Procedures. Prentice-Hall, New Jersey.

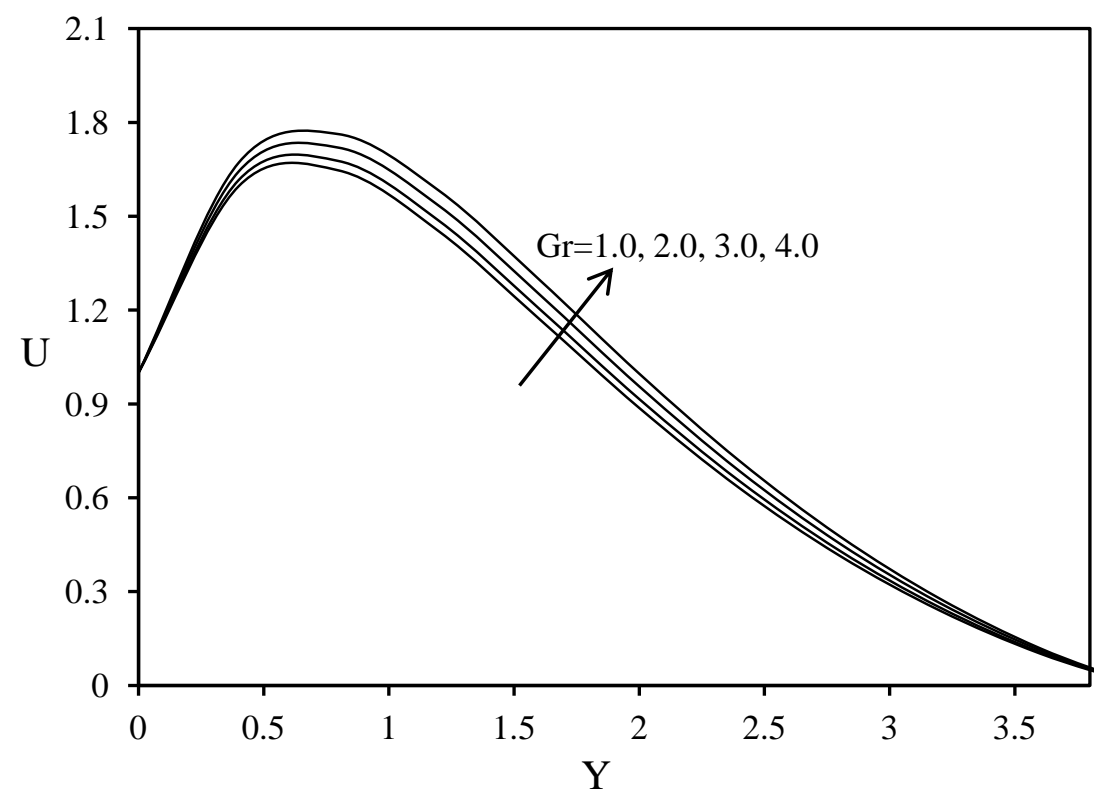

Fig 2: Velocity profiles for different values of $G r$

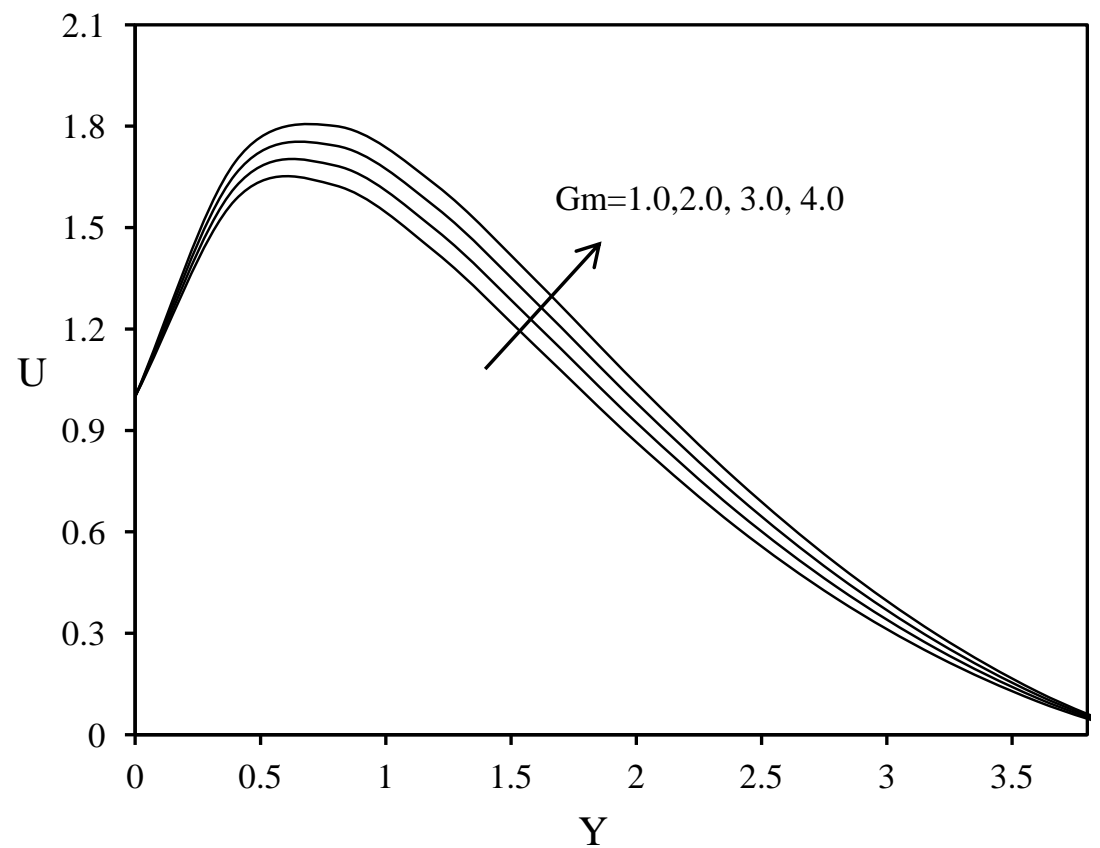

Fig 3: Velocity profiles for different values of $\mathbf{G m}$ 


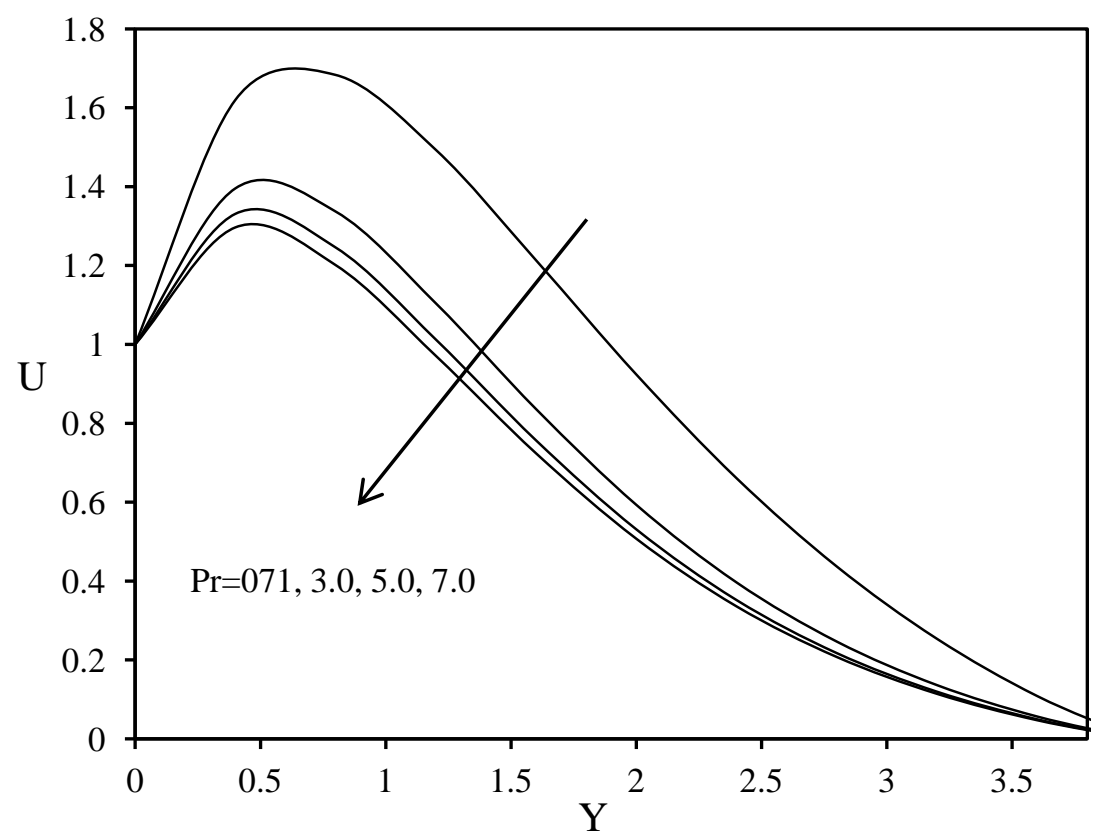

Fig 4: Velocity profiles for different values of $\mathbf{P r}$

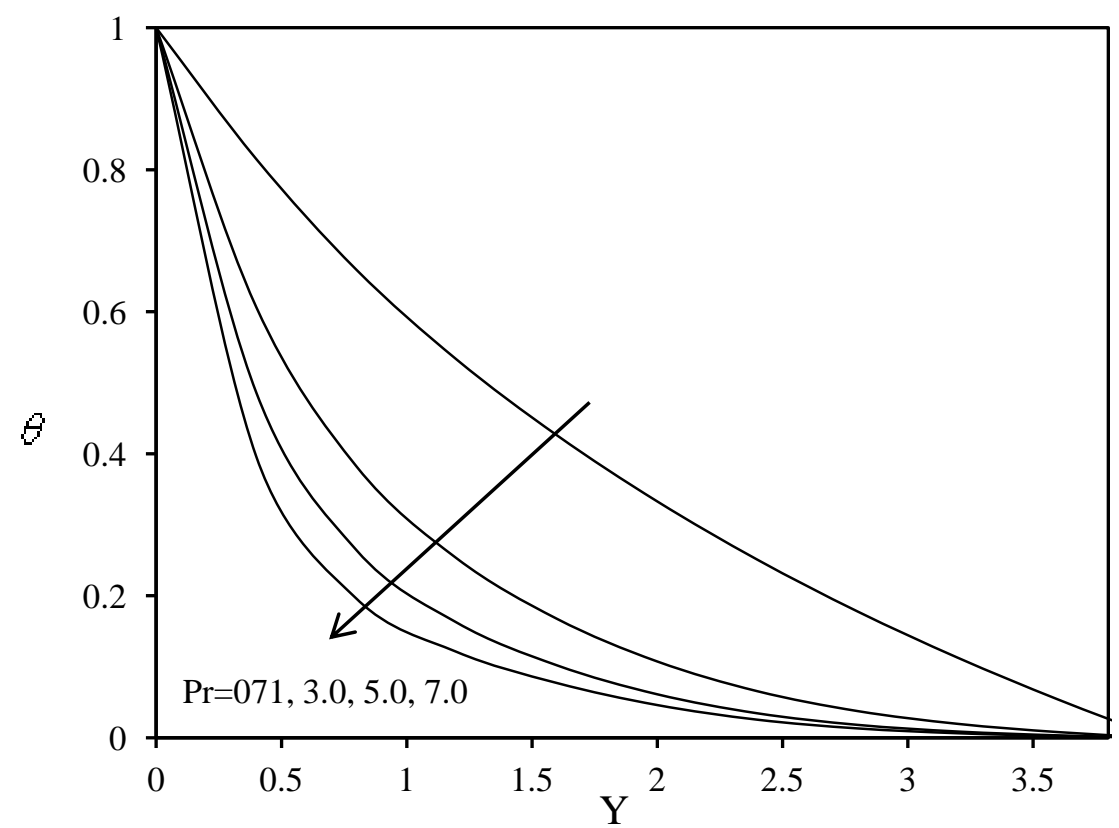

Fig 5: Temperature profiles for different values of $\mathbf{P r}$ 


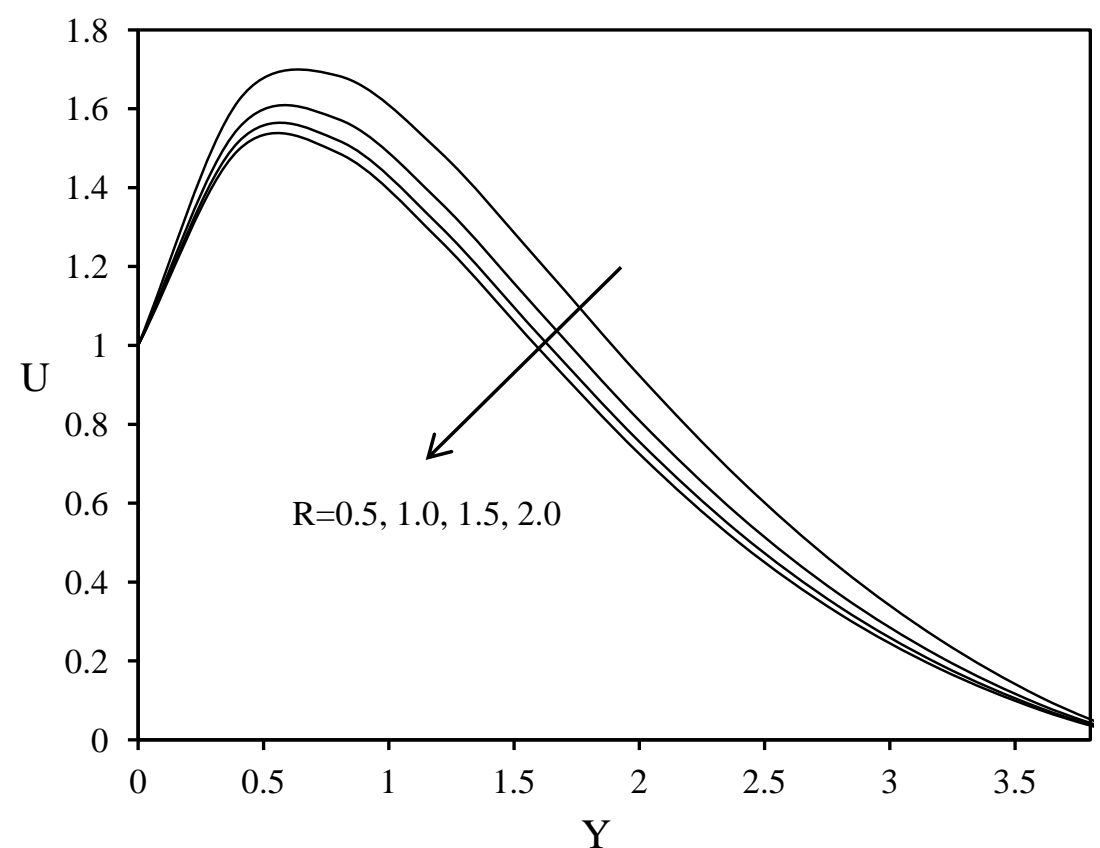

Fig 6: Velocity profiles for different values of $\boldsymbol{R}$

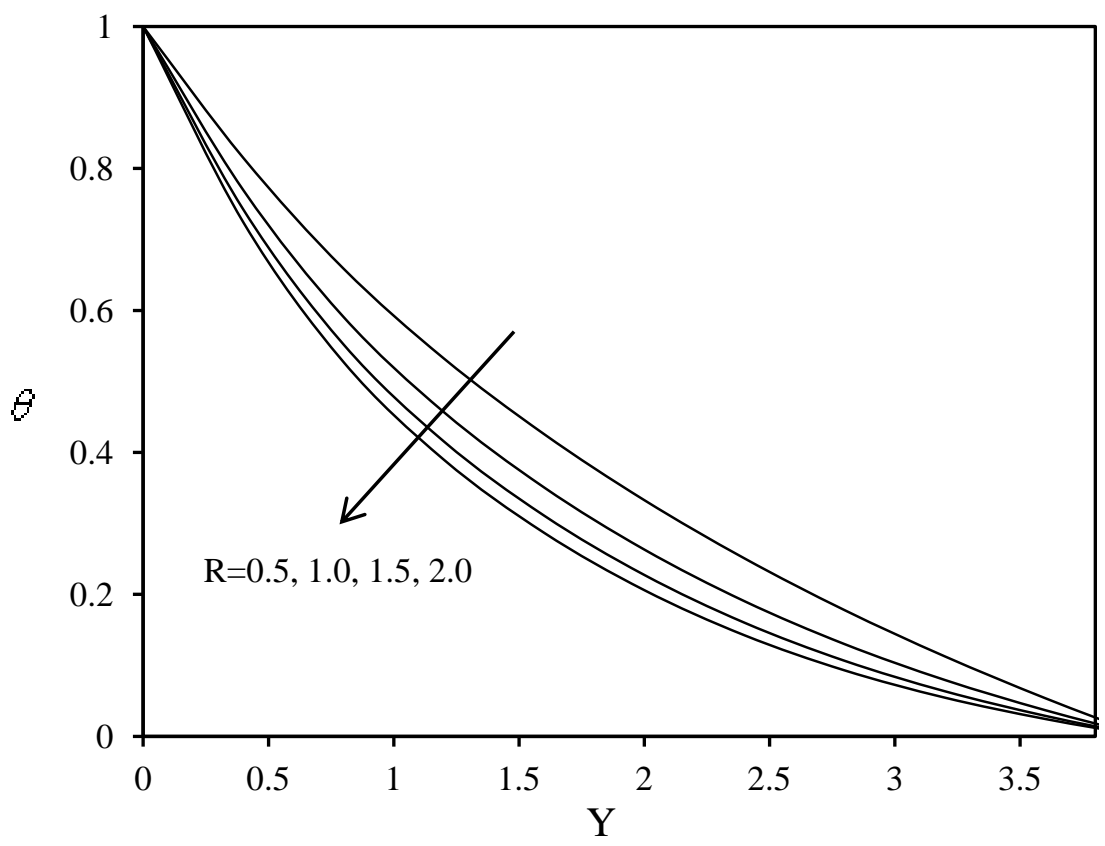

Fig 7: Temperature profiles for different values of $\boldsymbol{R}$ 


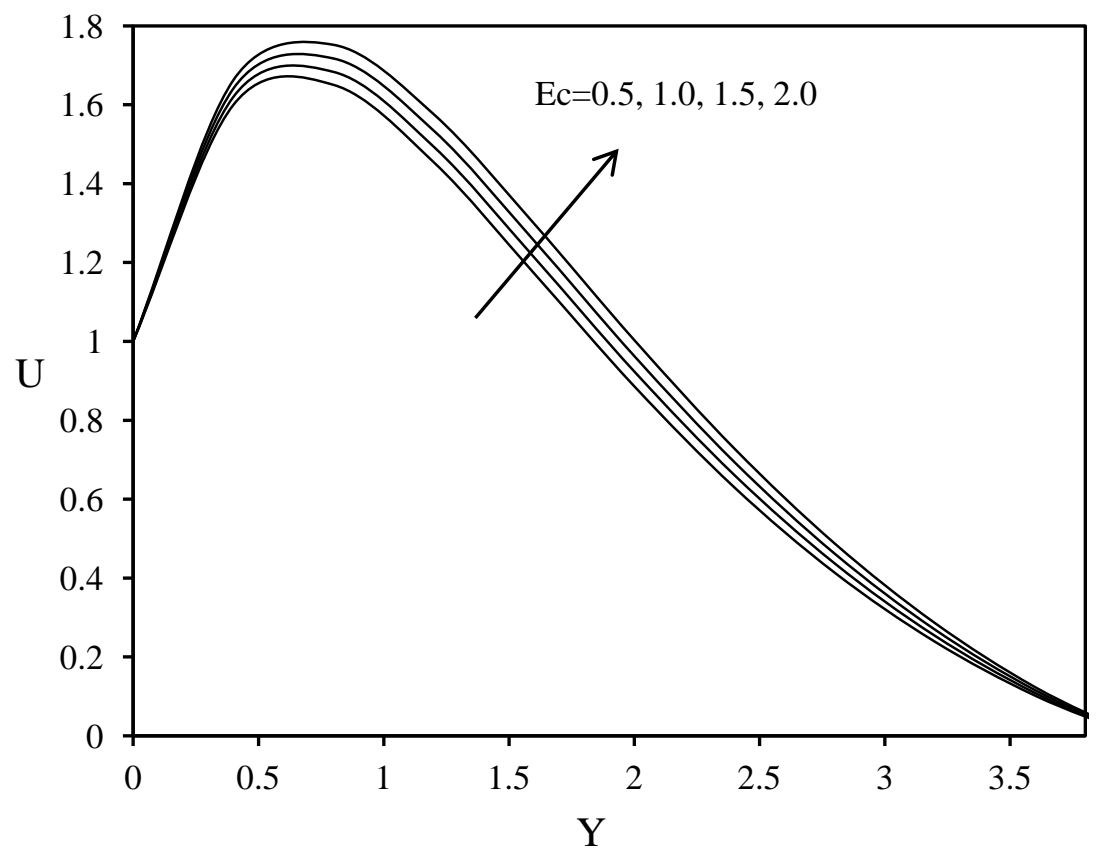

Fig 8: Velocity profiles for different values of $\boldsymbol{E} \boldsymbol{c}$

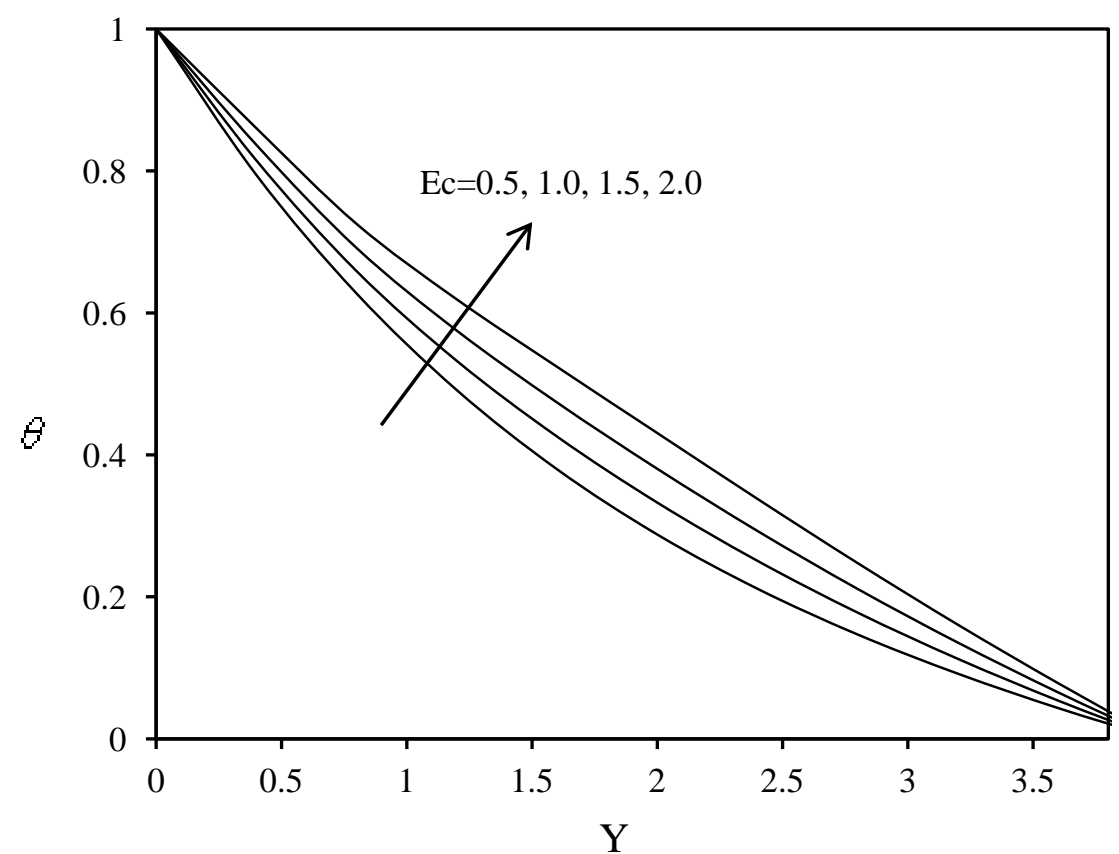

Fig 9: Temperature profiles for different values of $\boldsymbol{E} \boldsymbol{C}$ 


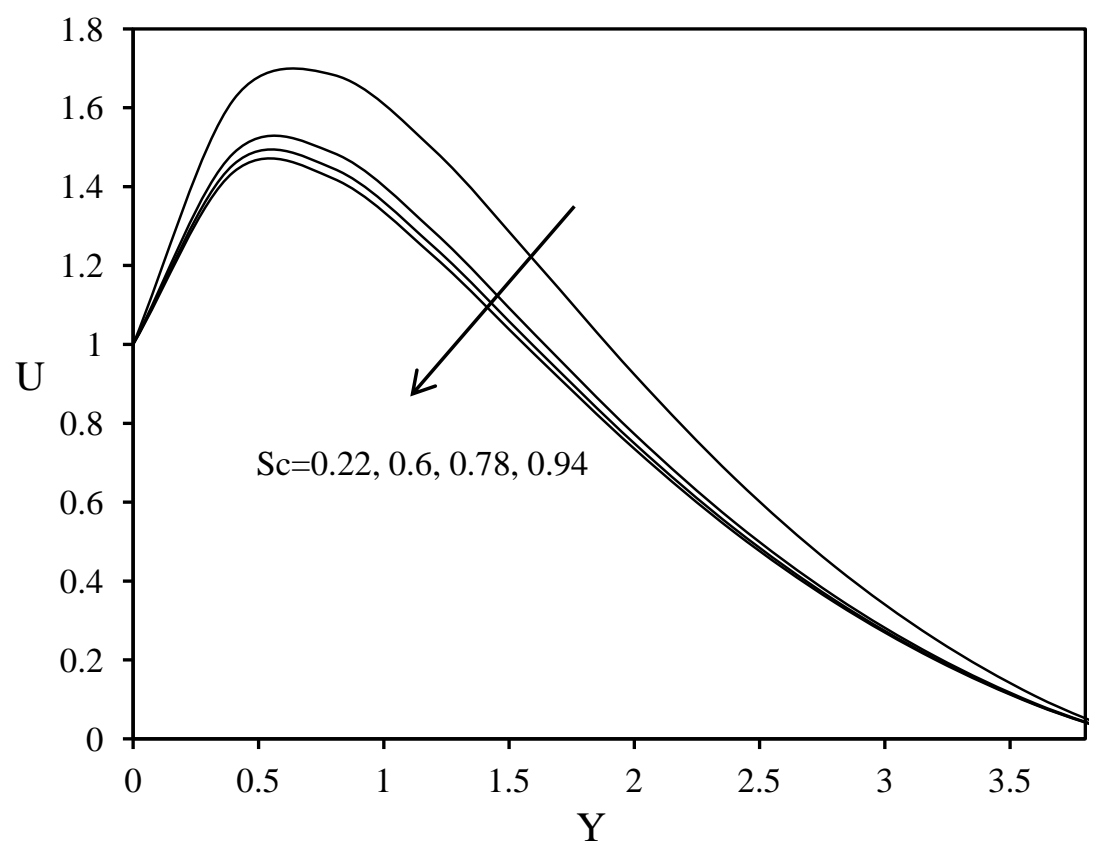

Fig 10: Velocity profiles for different values of $S \boldsymbol{C}$

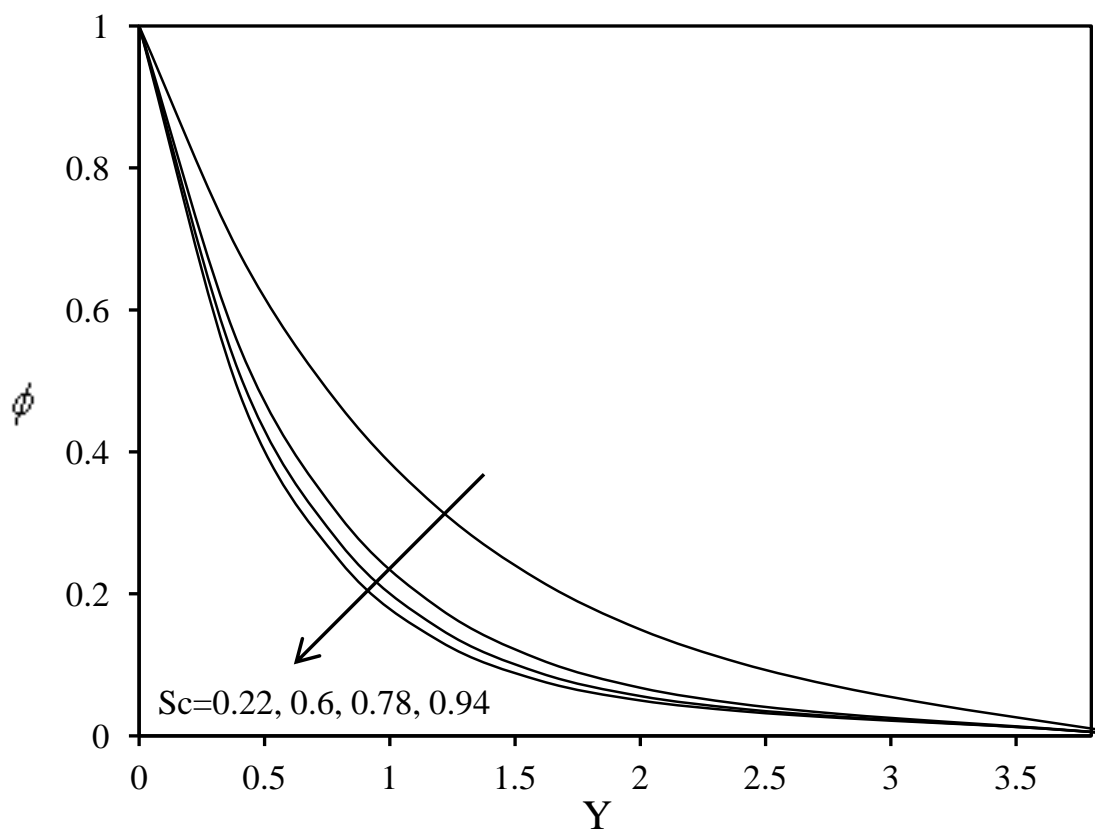

Fig 11: Concentration profiles for different values of $S c$ 


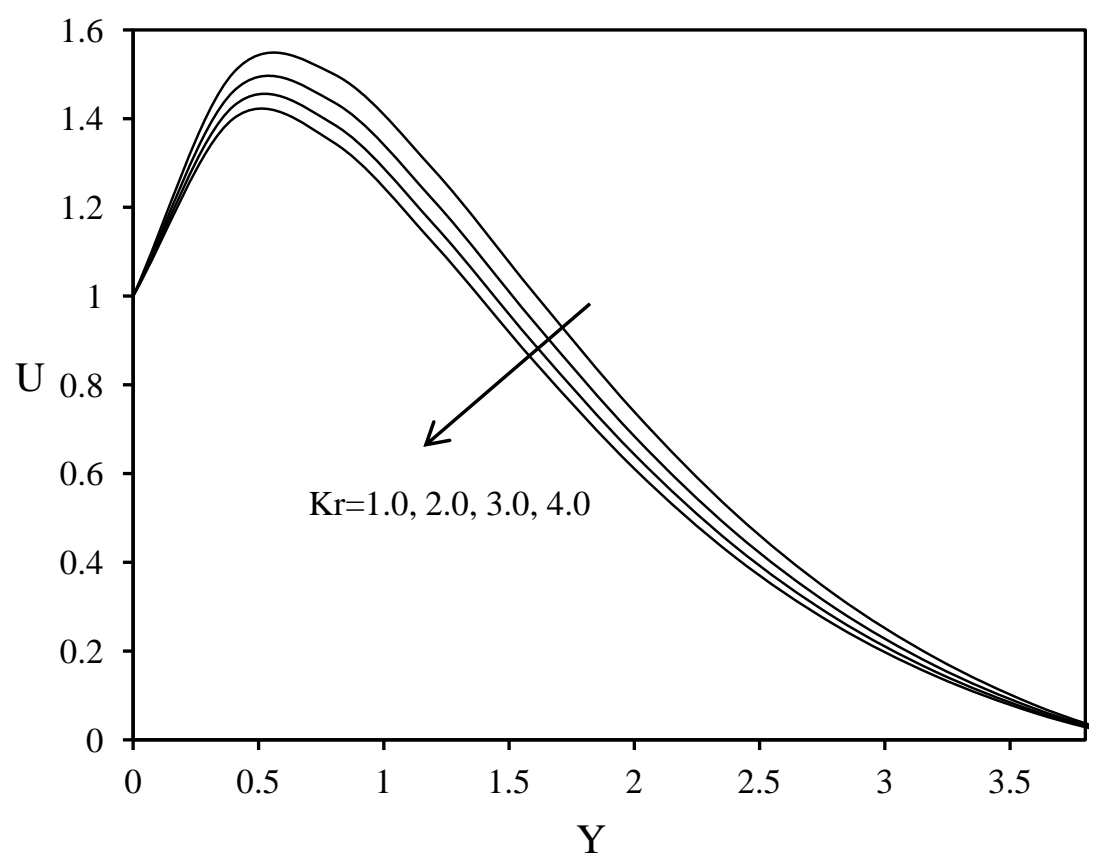

Fig 12: Velocity profiles for different values of $\boldsymbol{K} \boldsymbol{r}$

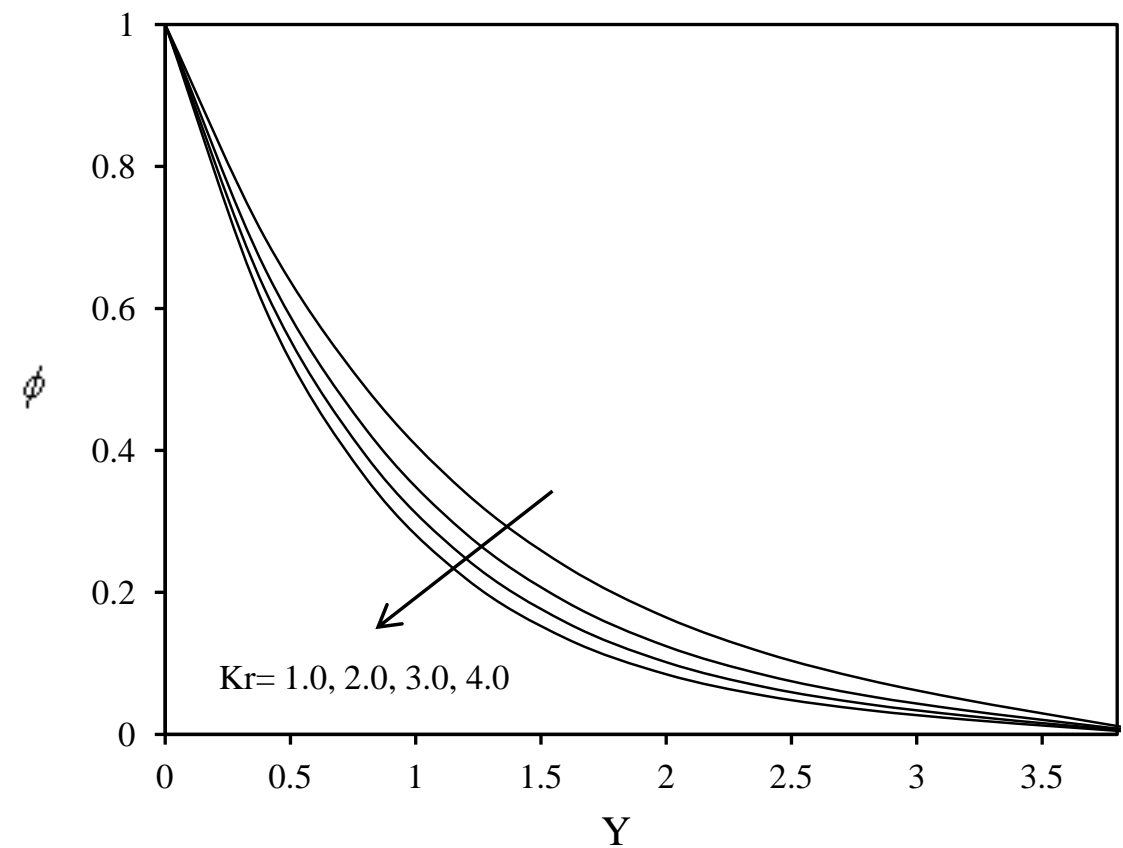

Fig 13: Concentration profiles for different values of $\boldsymbol{K} \boldsymbol{r}$ 